Center for

Mathematical Economics

Working Papers

April 2017

\title{
Asymmetric Dominance Effect with Multiple Decoys for Low- and High-Variance Lotteries
}

Oktay Sürücü, Sonja Brangewitz and Behnud Mir Djawadi

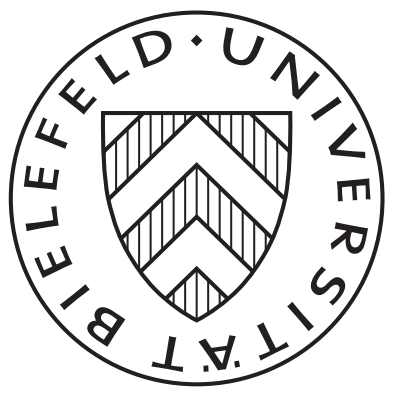




\title{
Asymmetric Dominance Effect with Multiple Decoys for Low- and High-Variance Lotteries
}

\author{
Oktay Sürüc $\ddot{u}^{1}$, Sonja Brangewitz ${ }^{2}$ and Behnud Mir Djawadi ${ }^{3}$ \\ ${ }^{1}$ Center for Mathematical Economics, Bielefeld University, \\ Postfach 100131, 33501 Bielefeld, Germany \\ ${ }^{2}$ Department of Economics, Paderborn University, \\ Warburger Str. 100, 33098 Paderborn, Germany \\ ${ }^{3}$ Department of Management, Paderborn University, \\ Warburger Str. 100, 33098 Paderborn, Germany
}

Email: oktay.surucu@uni-bielefeld.de|sonja.brangewitz@wiwi.upb.de|behnud.mir.djawadi@upb.de

April 28, 2017

\begin{abstract}
The asymmetric dominance effect refers to the phenomenon according to which the choice probability of an alternative increases when an inferior alternative - the decoy - is included into the choice set. The objective of this experimental study is twofold. First, we investigate the asymmetric dominance effect on two-outcome lotteries with almost equal expected values. We find that the impact of a decoy on low-variance lotteries $(L V L \mathrm{~s})$ is much higher than on high-variance lotteries $(H V L \mathrm{~s})$. Second, we examine the asymmetric dominance effect in the presence of two decoys. While the asymmetric dominance effect persists when the choice set includes two decoys, the effect is not always further enhanced compared to the setting with one decoy and again much stronger for $L V L$ s than for $H V L$ s. Controlling for subjects' degrees of risk aversion, we find support for consistency between individual risk preferences and choice behavior among the lotteries. However, we observe decoy effects of equal strength irrespective of the subjects' degree of risk aversion. Thus, our analysis indicates that to a substantial extent the presence of decoys subtly makes decision-makers choose against their risk preferences by favoring lotteries that entail risks contrary to their elicited individual risk-taking profile.
\end{abstract}

Keywords: Asymmetric dominance effect, decoy effects, multiple decoys, risk aversion, individual decision making, experimental economics

JEL Classification Numbers: C91, D12, D83

\section{Acknowledgments}

This work was partially supported by the German Research Foundation (DFG) within the Collaborative Research Centre "On-The-Fly Computing" (SFB 901). 


\section{Introduction}

There is overwhelming evidence that the structure of a choice set can influence agents' choices: that is, choices are context-dependent. The asymmetric dominance effect, first demonstrated by Huber et al. (1982), is among the most studied context-dependent behaviors. This effect refers to the systematic violation of rationality induced by the introduction of an inferior alternative to a choice set. More specifically, it can be described as the phenomenon according to which the choice probability of an alternative might increase when an inferior alternative is included in the choice set. Consider, for example, a binary choice set consisting of two alternatives, $A$ and $B$, that are characterized by two attributes. Suppose $A$ is better in the first attribute and $B$ is better in the second. In a typical experimental study both $A$ and $B$ are chosen. Now consider the introduction of a third alternative that is dominated by one of the two alternatives, say by $A$, as illustrated in Figure 1. According to the rationality principle, this newly introduced alternative, which is referred to as decoy, does not have any impact on choices. It should basically be ignored due to being dominated by another alternative in the choice set. However, evidence shows that there is a significant increase in the market share of the dominating alternative, hence the name target.

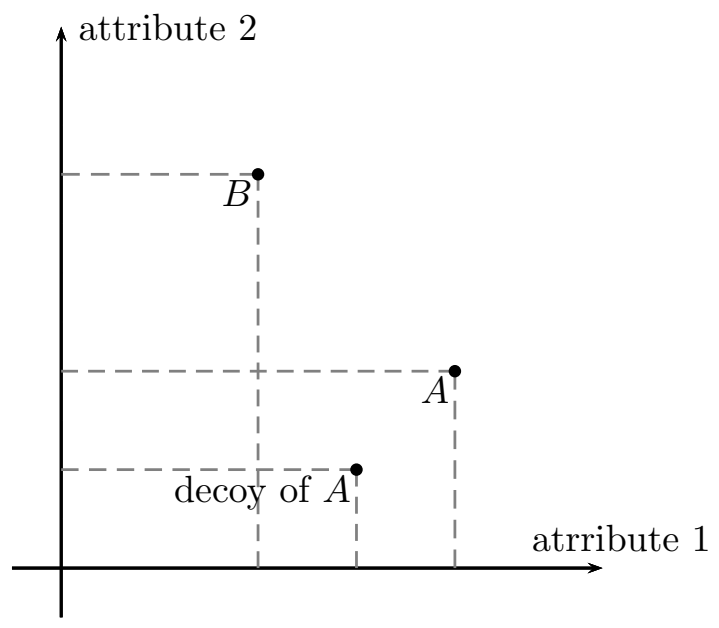

Figure 1: Choice Set with a Decoy

This systemic violation of rationality has been studied extensively in the literature on psychology, marketing, consumer behavior and decision making, and replicated using many different experimental settings. The effect has been observed when subjects are given real incentives (Herne, 1998, 1999; Simonson and Tversky, 1992); when choice sets consist of non-market alternatives (Bateman et al., 2008), policy alternatives (Herne, 1997), gambles (Herne, 1999; Wedell, 1991) or investment alternatives (Schwarzkopf, 2003); when the experimental design is withinsubject (Lehmann and Pan, 1994; Simonson, 1989; Wedell and Pettibone, 1996) or betweensubject (Dhar and Glazer, 1996; Highhouse, 1996) and even when the subjects are hummingbirds (Bateson et al., 2003), gray jays (Shafir et al., 2002), túngara frogs (Lea and Ryan, 2015) or amoeboid organisms (Latty and Beekman, 2011). Furthermore, in a field experiment conducted in a grocery store, Doyle et al. (1999) have shown that the asymmetric dominance effect plays an important role in actual markets.

After its demonstration by Huber et al. (1982), several explanations for why the asymmetric dominance effect occurs have been put forward. Among the most commonly tested explanations is the range-frequency theory by Parducci (1974). This theory points out that the introduction of a decoy has two impacts on the choice set: first, it extends the range of the attribute on which the target is weakest, and second, it increases the frequency of the other attribute on which the 
target is superior. For example, thanks to the decoy, the choice set illustrated in Figure 1 has a wider range on attribute 2 and a higher frequency on attribute 1 with respect to the choice set without the decoy. According to the range-frequency theory, the extension in the range causes the distance on attribute 2 between the target and its competitor to be perceived as smaller than it would have been without the decoy. The frequency increase instead tends to spread out the perceived distance on attribute 1 between the target and its competitor. Therefore, the introduction of a decoy makes the target look better in comparison to the competitor by increasing its perceived attractiveness and thus causes the asymmetric dominance effect. To distinguish between the range and the frequency explanations, Huber et al. (1982) use three different types of decoys: (i) the $r$-decoy which only extends the relevant range without changing frequency, (ii) the $f$-decoy which only increases the relevant frequency without touching to range, (iii) the $r f$-decoy which combines $r$ - and $f$-decoys. Figure 2 illustrates a choice set, where $A$ is the target and $A_{r}^{\prime}, A_{f}^{\prime}$ and $A_{r f}^{\prime}$ are $r$-, $f$ - and $r f$-decoys, respectively. Huber et al. (1982) find no significant results for frequency increase but do for the range extension hypothesis. However, the results seem to suggest that a simple range extension explanation alone is not sufficient. Heath and Chatterjee (1995) report a meta-analysis that supports the range extension explanation and demonstrates that larger range extensions produce larger increases in the target's share.

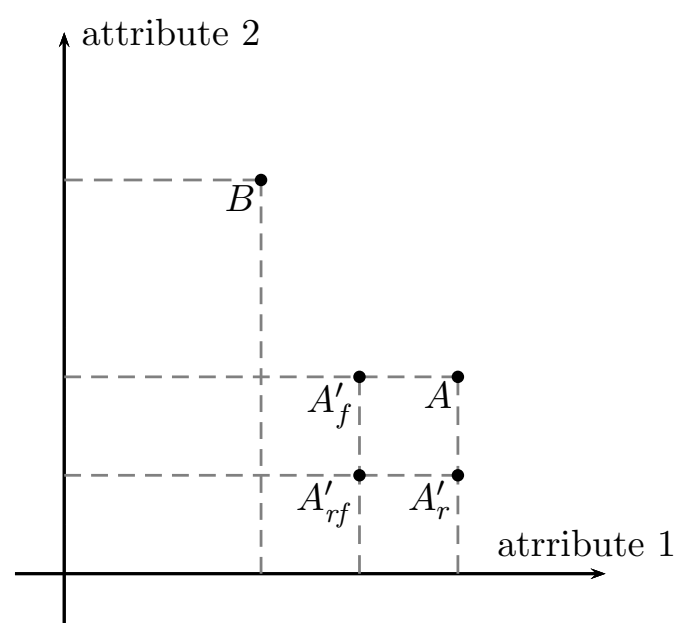

Figure 2: Choice Set with Different Types of Decoys

Wedell (1991) extends the analysis of Huber et al. (1982) to the environment where choice alternatives are two-outcome lotteries. He shows that the asymmetric dominance effect is highly significant and the results support both range and frequency explanations. However, there is no significant change when different types of decoys are used. On account of this observation, Wedell (1991) claims that the asymmetric dominance effect is best explained by agents' use of the dominance heuristic. According to this explanation, agents employ a decision strategy in which they first look for a dominance relation between alternatives and choose the dominating alternative if such a relation exists. This hypothesis is also supported by the findings of a Magnetic Resonance Image (MRI) study that show people avoid trade-off contrast and search for dominance (Hedgcock and Rao, 2009). Another experimental investigation of the asymmetric dominance effect where two-outcome lotteries are used as choice alternatives is Herne (1999). In this study, only $r f$-decoys are considered and subjects are given monetary incentives. ${ }^{1}$ The

\footnotetext{
${ }^{1}$ In Herne (1999), subjects were given a participation fee plus the outcome of a randomly chosen choice task. On the other hand, the incentivization mechanism used in Wedell (1991) was to give subjects course credits for their participation.
} 
results indicate that the effect persists when real incentives are used.

The economic literature proposes several choice models that accommodate the asymmetric dominance effect. Masatlioglu et al. (2016) and Lleras et al. (2016) consider the situation, in which agents are limited in their ability to consider all alternatives and concentrate only on a subset of a given choice set. Agents choose the normatively best alternative in this subset. These two models are compatible with the asymmetric dominance effect if the target, but not the competitor, belongs to the set considered by agents. Ok et al. (2014) propose a referencedependent model in which given a choice set, agents choose by maximizing utility over those alternatives that Pareto dominate a reference alternative. Lombardi (2009) axiomatizes a model where agents select alternatives that are maximal according to a fixed but not necessarily complete preference relation. If the resulting maximal set has more than one element, agents remove those alternatives whose lower contour set is strictly contained in that of another maximal alternative. This model accommodates the asymmetric dominance effect only if agents are indifferent between the target and the competitor or these two alternatives are not comparable according to the agents' preference relation. The reference-dependent model proposed by Barbos (2010) concerns the situation in which choices are made across exogenously given categories. Agents make choices as if they first evaluate each category and then choose by maximizing utility over their favorite category. The model is compatible with the asymmetric dominance effect under the assumption that agents perceive the target and the decoy as belonging to a category and the competitor to another. de Clippel and Eliaz (2012) introduce a model where agents' choices are the cooperative solution to a bargaining problem between two selves. This model accommodates the asymmetric dominance effect except when an $f$-decoy is used.

This article aims to shed light on some uninvestigated issues regarding the asymmetric dominance effect when choice sets consist of lotteries. We first address the question of whether the introduction of a decoy has a different impact on low-variance lotteries ( $L V L s)$ with a high probability of low winnings than on high-variance lotteries $(H V L \mathrm{~s})$ with a low probability of high winnings. Secondly, we explore the effect in the presence of two decoys. The literature on the asymmetric dominance effect concentrates only on situations in which there exists a single decoy, and leaves open the questions whether the effect boosts or diminishes, and whether $L V L \mathrm{~s}$ and $H V L$ s are affected in the same manner in the presence of multiple decoys. Our aim is to close this gap in the literature. Furthermore, we examine the question that is brought along by the investigation of the asymmetric dominance effect on lotteries differing in their risk components: How is the effect related to risk aversion?

In this article, we experimentally study and analyze individual choice behavior for twooutcome lotteries. In addition to the control, we have four different treatments. In the first two treatments, subjects face a choice decision from a set of three lotteries: an $L V L$, an $H V L$ and a decoy (an $r$-decoy or an $f$-decoy). The choice sets of the other two treatments include a $L V L$, a $H V L$ and two decoys (two $r$-decoys or two $f$-decoys). Finally, in our control treatment, choice sets are decoyless: they consist of a $L V L$ and a $H V L$. Each treatment ends with a questionnaire that is served to elicit subjects' risk preferences.

Our analysis suggests that the asymmetric dominance effect is stronger when the target is an $L V L$ than when it is an $H V L$. The effect persists also when the choice set includes two decoys. It seems that the effect is generated by the first decoy and the second one does not have any further impact on it. However, there is one exception: When the choice set includes two $r$-decoys that target an $H V L$, the choice probability of the target increases significantly compared to the case with a single $r$-decoy. Hence, only in this specific case does the secondary decoy boost the asymmetric dominance effect. Interestingly, this observed increase in the choice probability of an $H V L$ target does not invalidate our first result in the presence of two decoys. That is, the asymmetric dominance effect with two decoys is stronger when the target is an $L V L$ than when it is an $H V L$. Furthermore, we also control for the subjects' risk attitudes and 
obtain strong evidence that the asymmetric dominance effect does not induce any inconsistency in risk preferences. More specifically, the asymmetric dominance effect does not intervene the trend that the more risk averse subjects are, the more the less risky alternative $L V L$ is chosen. Finally, our analysis indicates that subjects exhibit the asymmetric dominance effect irrespective of their degree of risk aversion, and there seems to exist no correlation between the degree of risk aversion and the strength of the effect.

The organization of the paper is as follows. Section 2 presents the experimental design and procedure. Section 3 explains the hypotheses. Section 4 gives the results. Finally, Section 5 concludes with a discussion.

\section{Experiment}

\subsection{Experimental Design}

In a computerized laboratory setting, we asked subjects to choose between a number of twooutcome lotteries, each characterized by an amount to win (in our experimental currency unit, "Taler") and its associated probability. The second outcome was always 0 Taler. The lotteries were displayed by means of pies including an explanatory text. An example is shown in Figure 3.

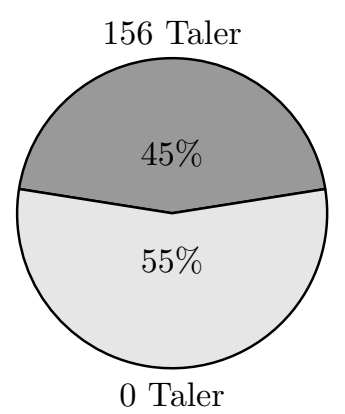

With this lottery you have a $45 \%$ chance to win 156 Taler, nothing otherwise.

Figure 3: Graphical Illustration of a Lottery

The experiment involved 4 main treatments, each of which included 54 choice tasks. In addition, one control treatment with 27 choice tasks was conducted. In the control treatment (T0), the choice sets consisted of two lotteries with almost equal expected values. An example of a choice set used in $\mathbf{T 0}$ is the following: A lottery, say $A$, that gives 156 Taler with a probability of 0.45 and a second lottery, say $B$, that gives 280 Taler with a probability of 0.25 . In other words, subjects were asked to choose between a low-variance lottery $(L V L)$ with high probability of low winnings (lottery $A$ ) and a high-variance lottery $(H V L)$ with low probability of high winnings (lottery $B$ ). These two lotteries (together with some others) are depicted in Figure 4. The choice sets of treatments T1 and T2 consisted of three lotteries; two baseline lotteries used in treatment T0 and a decoy. The type of decoy differed between the two treatments. We used $r$-decoys in $\mathbf{T} \mathbf{1}$ and $f$-decoys in treatment $\mathbf{T} \mathbf{2}$. In half of the choice tasks (both in $\mathbf{T} \mathbf{1}$ and in T2), target lotteries were $L V L \mathrm{~s}$ and in the other half they were $H V L \mathrm{~s}$. Put differently, any two baseline lotteries from treatment $\mathbf{T 0}$ appeared in exactly two choice sets of treatments $\mathbf{T} \mathbf{1}$ and T2; each time the decoy was targeting a different lottery. For example, in treatment T1, the two baseline lotteries $A$ and $B$ used in treatment To (demonstrated in Figure 4) were juxtaposed to an $r$-decoy targeting lottery $A\left(A_{r}^{\prime}\right.$ in Figure 4$)$ in one choice task. In another, they were juxtaposed to an $r$-decoy targeting lottery $B\left(B_{r}^{\prime}\right.$ in Figure 4$)$. The same procedure of choice set construction applied also in treatment $\mathbf{T} 2$, but using $f$-decoys. That is, the two choice sets 
where lotteries $A$ and $B$ appeared were $\left\{A, B, A_{f}^{\prime}\right\}$ and $\left\{A, B, B_{f}^{\prime}\right\}$ (see Figure 4 ). In treatment T3, we used the same set of choice tasks from T1 but added a second $r$-decoy to each of them. Similarly, choice tasks of treatment $\mathbf{T} \mathbf{4}$ are constructed by adding a second $f$-decoy to the choice tasks of treatment T2. Hence, subjects of the last two treatments were asked to choose between four lotteries: two baseline and two decoy lotteries of the same type. Examples of choice sets from T3 (T4) are $\left\{A, B, A_{r}^{\prime}, A_{r}^{\prime \prime}\right\}$ and $\left\{A, B, B_{r}^{\prime}, B_{r}^{\prime \prime}\right\}\left(\left\{A, B, A_{f}^{\prime}, A_{f}^{\prime \prime}\right\}\right.$ and $\left.\left\{A, B, B_{f}^{\prime}, B_{f}^{\prime \prime}\right\}\right)$ which are illustrated in Figure 4.

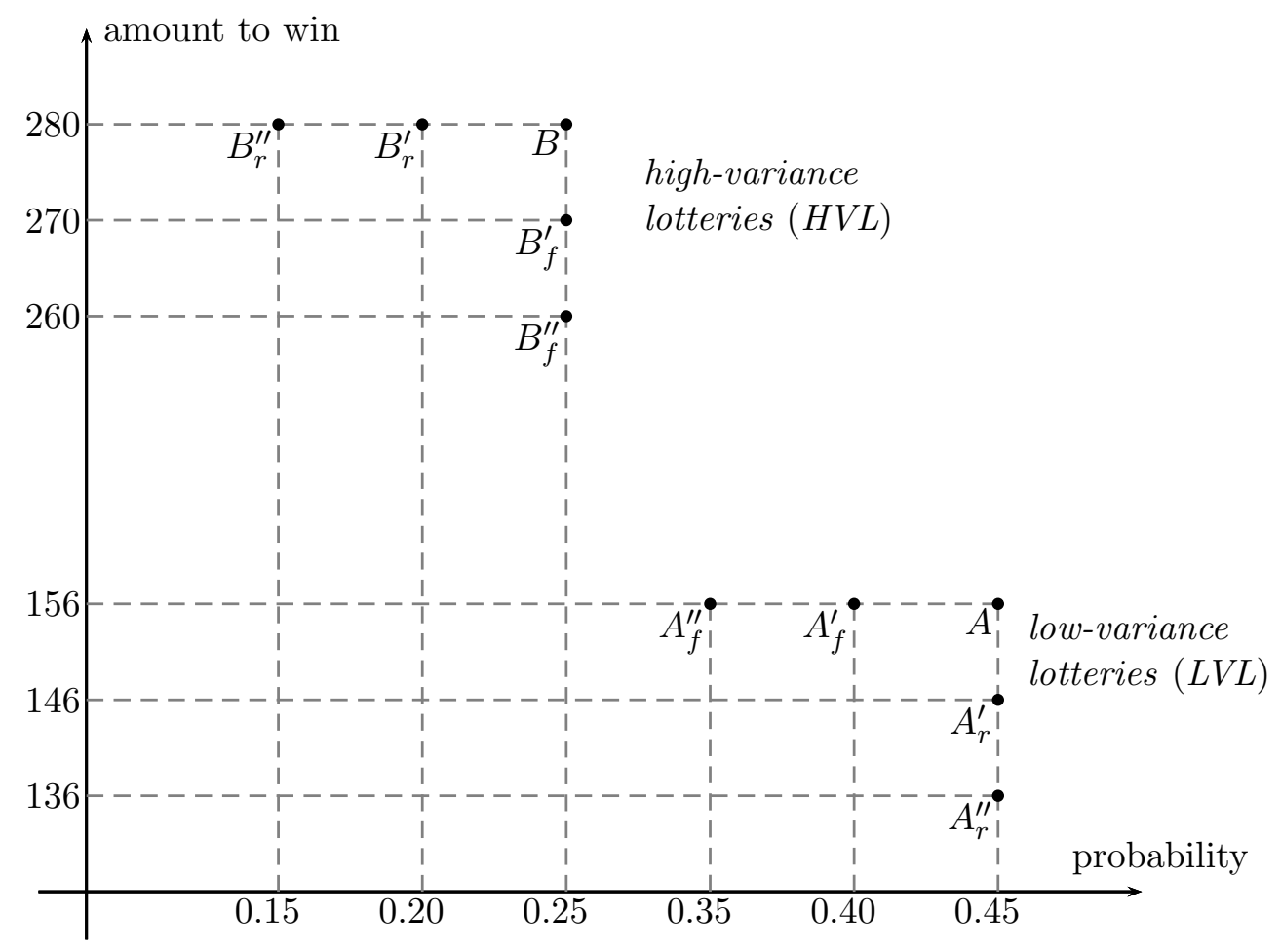

Figure 4: Illustration of Some Lotteries Used in the Experiment

Table 1 summarizes the choice set structures of the control (T0) and the 4 treatments (T1T4). The complete list of lotteries used in the experiment can be found in Table 8 in Appendix A. For internal reference we numbered the tasks from 1 to 27 when decoys target an $L V L$ and from 28 to 54 when decoys target an $H V L$. The order of all the 54 tasks in each treatment and the display order of lotteries in each choice task was randomized anew for each subject. In each treatment, after the main experiment, subjects were asked to answer a questionnaire which was

\begin{tabular}{|l|c|c|c|c|c|}
\hline & T0 & T1 & T2 & T3 & T4 \\
\hline number of alternatives & 2 & 3 & 3 & 4 & 4 \\
\hline number of decoys & 0 & 1 & 1 & 2 & 2 \\
\hline decoy type & & $r$-decoy & $f$-decoy & two $r$-decoys & two $f$-decoys \\
\hline \multirow{2}{*}{ tasks 1-27 } & $L V L$, & $L V L, H V L$ & $L V L, H V L$ & $L V L, H V L$ & $L V L, H V L$ \\
& $H V L$ & $L V L_{r}^{\prime}$ & $L V L_{f}^{\prime}$ & $L V L_{r}^{\prime}, L V L_{r}^{\prime \prime}$ & $L V L_{f}^{\prime}, L V L_{f}^{\prime \prime}$ \\
\cline { 2 - 7 } & & $L V L, H V L$ & $L V L, H V L$ & $L V L, H V L$ & $L V L, H V L$ \\
& & $H V L_{r}^{\prime}$ & $H V L_{f}^{\prime}$ & $H V L_{r}^{\prime}, H V L_{r}^{\prime \prime}$ & $H V L_{f}^{\prime}, H V L_{f}^{\prime \prime}$ \\
\hline
\end{tabular}

Table 1: Choice Set Structures 
divided into two parts. In the first part, risk preferences were elicited by using the incentivized lottery choice framework by Dohmen et al. (2010), followed by an unpaid second part with questions on the socio-economic background of the participants: e.g., age, gender, field of study, etc.

\subsection{Experimental Procedure}

The experiment was programmed in C++ using a z-tree interface (Fischbacher, 2007) and conducted at the Business and Economic Research Laboratory (BaER-Lab) at Paderborn University, Germany. Subjects were recruited by the online recruiting system ORSEE (Greiner, 2015) from a pool of approximately 2,800 voluntary students of Paderborn University from different fields of study, who are enrolled as prospective participants in economic experiments. Upon entering the laboratory, subjects were randomly assigned to visually isolated computer terminals. The instructions were distributed and subjects were asked to read them on their own. In order to avoid misunderstandings, they were allowed to ask questions individually to an experimenter.

We ran one session for each treatment with 28 subjects. Overall, we collected 6,804 observations from 140 subjects across 5 sessions. On average, each session lasted about 75 minutes, including the time used for reading the instructions and paying the subjects.

At the end of each treatment, a choice task was randomly chosen and subjects played the lottery that they had chosen in that task. Note that the task was identical for all subjects in a session. However, due to the order randomization, different subjects faced the chosen task at different positions in the course of the experiment. Subjects played the lottery that they had chosen by drawing a ball from an urn that corresponded to their lottery choice in the task. The experimental currency unit, Taler, was converted into Euros at the rate of 0.05 and subjects were paid according to the outcome of their chosen lottery. On top of that, in order to incentivize choice decisions of the questionnaire, which we used to elicit risk preferences, two subjects were randomly chosen and paid for one random decision row of their choice table. Average earnings per subject were 8.54 Euro (inclusive of a 4 Euro participation fee).

\section{Research Hypotheses}

Our aim in this article is to explore some issues that have not been investigated in the asymmetric dominance effect literature so far. We start with addressing the question whether the asymmetric dominance effect differs depending on the riskiness of a target lottery. More precisely, given two lotteries of the same expected value, we examine whether the effect is strongest when the target is the lottery with lower variance $(L V L)$ or the other one with higher variance $(H V L)$. There is clear evidence that people, in general, are risk averse and therefore choose the former over the latter in absence of a decoy. For example, in the first stage of preference reversal experiments, where subjects are asked to make a choice, a substantial fraction of them is shown to prefer $L V L$ s over HVLs (see, for example, Lichtenstein and Slovic, 1971; Slovic and Lichtenstein, 1983). Given this general tendency, we expect the asymmetric dominance effect to be stronger when the target is an $L V L$ than when it is an $H V L$.

Hypothesis 1. The asymmetric dominance effect is stronger when the target is a low-variance lottery than when it is a high-variance lottery (TO versus $\mathbf{T 1}, \mathbf{T O}$ versus $\mathbf{T} 2)$.

In our next hypothesis, we turn our attention to the impact of a secondary decoy on the asymmetric dominance effect. There are two prominent explanations for why we observe this effect in the presence of a single decoy: the range-frequency theory by Parducci (1974) and the dominance heuristic. The range-frequency explanation basically attributes the effect to the fact that the introduction of a decoy extends the range of the dimension on which the target is weakest 
and/or it increases the frequency of the dimension on which the target is superior. According to this explanation, a secondary decoy would boost the effect since it further extends the range and/or further increases the frequency. On the other hand, the dominance heuristic explanation attributes the effect to the dominance relationship between the alternatives of a choice set. That is, agents employ a decision strategy in which they first look for a dominance relation between alternatives and choose the dominating alternative if such a relation exists. According to this explanation, a secondary decoy would not change choice behaviors since it does not change the dominance relationship among the choice alternatives. Hence, our investigation has the potential to assess these two prominent explanations. In our second hypothesis, we first test the existence of the asymmetric dominance effect with two decoys and then compare it to the case with a single decoy.

\section{Hypothesis 2.}

(a) The asymmetric dominance effect exists when there are two decoy lotteries (T3 and T4).

(b) The addition of a second decoy has a further impact on the asymmetric dominance effect ( $\boldsymbol{T} 3$ versus $\mathbf{T} 1, \mathbf{T} 4$ versus $\mathbf{T} 2)$.

Considering a secondary decoy gives rise to a question similar to the one investigated by Hypothesis 1: Is the impact of the asymmetric dominance effect with two decoys different on $L V L$ s than on $H V L s$ s? We expect a similar result as in Hypothesis 1, and therefore our next hypothesis is:

Hypothesis 3. The asymmetric dominance effect with two decoys is stronger when the target is a low-variance lottery than when it is a high-variance lottery (TO versus $\mathbf{T} 3$, TO versus $\mathbf{T} 4)$.

Since all decisions are to be made under risk, individual preferences towards risk must be addressed and choice behavior triggered by the degree of risk aversion disentangled from possible asymmetric dominance effects. According to Frederick (2005), risk preferences are strongly tied to a person's cognitive ability and therefore constitute an important determinant when making risky decisions. The relation between risk and behavior has also been examined in many experimental studies, showing that subjects' risk attitudes often influence their behavior in games (see e.g. Straub, 1995, Schmidt et al., 2003, or Goeree et al., 2003). We are therefore interested in how the asymmetric dominance effect is related to risk preferences. Assuming consistency between risk attitudes and choice behavior, we expect that the share of choosing the $L V L$ increases with the degree of risk aversion. Therefore, we derive that the strength of the asymmetric dominance effect will be moderated by risk preferences. Our corresponding hypothesis is:

Hypothesis 4. The strength of the asymmetric dominance effect varies with individual risk preferences.

\section{Experimental Results}

The asymmetric dominance effect can be tested in two ways. The first one is used in betweensubject experimental designs and is run by comparing choice distributions between two sets: a set consisting of two baseline alternatives and another that includes the same two alternatives together with a decoy. A significant increase in the proportion of subjects choosing the target alternative indicates the asymmetric dominance effect. The second way, which is used in withinsubject design experiments, is to compare choice distributions between two sets, each of which includes the same two baseline alternatives but a decoy targeting a different alternative. The switch of choice from the target of one set to the target of the other set indicates the asymmetric 
dominance effect. We utilize both techniques in our analysis. The comparison of observations from treatment $\mathbf{T 0}$ with any other treatment gives the between-subject tests. The comparison of observations from the first half of the tasks with those from the second half in treatments $\mathbf{T} \mathbf{1}$, T2, T3 and T4 gives the within-subject tests.

Table 2 shows the choice distribution for each treatment. As mentioned earlier, in treatments T1, T2, T3 and T4 the decoys are always targeting an $L V L$ in the first half of the tasks (from 1 to 27 ) and an $H V L$ in the second half (from 28 to 54). The results from these treatments are presented in Table 2 as two-by-two contingency tables. For each contingency table, the upper left and lower right cells represent consistent choices (either lotteries $L V L$ or $H V L$ are chosen under both decoy conditions). The upper right and lower left cells represent inconsistent patterns of choice: the upper right cell shows the frequency of choosing $L V L$ when the decoy favors an $L V L$ and choosing $H V L$ when the decoy favors an $H V L$. Thus, the upper right cell corresponds to the asymmetric dominance effect. In Table 2, we exclude the observations in which a decoy lottery is chosen. There are 23 such observations in treatment $\mathbf{T 1}, 6$ in T2, 30 in T3 and 27 in T4. In total, 86 out of 3024 observation pairs were excluded (2.8\%). This strong tendency to avoid choosing a decoy alternative indicates that subjects can easily identify them.

Table 2 reveals that we successfully replicate the classical asymmetric dominance effect (with a single decoy) when choice alternatives are two-outcome lotteries. In treatment T1, where we use $r$-decoys, $17.7 \%$ of choices and in treatment $\mathbf{T} 2$, where we use $f$-decoys, $19.9 \%$ of choices exhibit a highly significant asymmetric dominance effect $\left(\mathcal{X}^{2}-\right.$ Test $(d f=1), \mathbf{T} 1: \mathcal{X}^{2}=155.9580$, $\left.p<0.001 ; \mathbf{T} 2: \mathcal{X}^{2}=164.9768, p<0.001\right)$.

In order to test Hypothesis 1, we need to compare the observations from treatment T0 to those from $\mathbf{T} \mathbf{1}$ and $\mathbf{T} \mathbf{2}$, separately. In treatment $\mathbf{T 0}$, the choice share of $L V L \mathrm{~s}$ and $H V L \mathrm{~s}$ are $56.7 \%$ and $43.3 \%$, respectively. First let us consider how the shares are distributed when choice sets include a decoy that targets an $L V L$ (tasks from 1 to 27 of treatment T1 and T2). As shown in Table 3, the choice share of $L V L \mathrm{~s}$ in treatment T1 is significantly higher than in T0 $(70.9 \%$ versus $56.7 \% ; \mathcal{X}^{2}$-Test $\left.(d f=1): 32.415, p<0.001\right)$ and the same holds true also for treatment T2 $\left(71.9 \%\right.$ versus $\left.56.7 \% ; \mathcal{X}^{2}-\operatorname{Test}(d f=1): 37.9328, p<0.001\right)$. On the contrary, when choice sets include a decoy that targets an $H V L$ (tasks from 28 to 54 of treatment T1 and T2), the choice share of $H V L$ s are not statistically different $\left(39.4 \%\right.$ versus $43.3 \%$ for treatment $\mathbf{T} 1 ; \mathcal{X}^{2}$ $\operatorname{Test}(d f=1): 2.2231, p>0.1$ and $42.1 \%$ versus $43.3 \%$ for treatment $\mathbf{T} 2 ; \mathcal{X}^{2}-\operatorname{Test}(d f=1): 0.279$, $p>0.1)$. In total, these results show that the asymmetric dominance effect is stronger when the target is an $L V L$ than when it is an $H V L$, and provide support for Hypothesis 1.

Now we turn our attention to the cases with two decoys. Table 2 reveals that $21.4 \%$ of choices in treatment $\mathbf{T} \mathbf{3}$ and $21.5 \%$ of those in treatment $\mathbf{T} \mathbf{4}$ account for the preference reversal described by the asymmetric dominance effect. Regardless of decoy types, we observe a significant choice distortion in the direction of target lotteries when choice sets include two decoy lotteries $\left(\mathcal{X}^{2}\right.$ $\operatorname{Test}(d f=1)$, T3: $\left.\mathcal{X}^{2}=136.9546, p<0.001 ; \mathbf{T} 2: \mathcal{X}^{2}=113.4453, p<0.001\right)$. Hence, we find support for Hypothesis 2(a). In order to investigate Hypothesis 2(b), we first examine the impact of a secondary $r$-decoy by comparing choice shares of treatment T1 and T3. The difference between the shares of the two treatments is significant $\left(\mathcal{X}^{2}(d f=3): 7.8981, p=0.048\right)$, meaning that the introduction of a secondary $r$-decoy boosts the asymmetric dominance effect. In order to gain more insight, we deepen our analysis by examining choice shares of $\mathbf{T} \mathbf{1}$ and $\mathbf{T} 3$ in the first and the second half of the tasks, separately. The comparison of choice shares of the tasks in which an $L V L$ is the target (tasks from 1 to 27$)$ yields no significant difference $\left(\mathcal{X}^{2}(d f=1): 1.8787\right.$, $p>0.1$ ). Differently put, the addition of a secondary $r$-decoy has no significant effect when the decoys target an $L V L$. On the other hand, the comparison of T1 and T3 in tasks in which an $H V L$ is the target (tasks from 28 to 54$)$ yields a significant difference $\left(\mathcal{X}^{2}(d f=1): 7.5652\right.$, $p=0.0060)$. This shows that including a secondary $r$-decoy that targets an $H V L$ significantly increases its choice probability, and hence gives rise to a boost in the asymmetric dominance 
effect. Secondly, we compare treatments $\mathbf{T} 2$ and $\mathbf{T} 4$ to see the impact of a secondary $f$-decoy. We find that the difference between the choice shares of the two treatments is not significant $\left(\mathcal{X}^{2}(d f=3): 2.9206, p>0.1\right)$. The same holds true when we examine the first and the second half of the tasks separately $\left(\mathcal{X}^{2}-\right.$ Test $(d f=1)$, tasks from 1 to $27: \mathcal{X}^{2}=0.1070, p>0.1$; tasks from 28 to 54: $\left.\mathcal{X}^{2}=0.0591, p>0.1\right)$. Thus, we conclude that the introduction of a secondary decoy lottery has an increasing impact on the asymmetric dominance effect only if the two decoys of the choice set are $r$-decoys and they target an $H V L$. Hence, we partially accept Hypothesis 2(b). As mentioned earlier, the two prominent explanations for why the classical asymmetric dominance effect (with a single decoy) is observed are the range-frequency theory by Parducci (1974) and the dominance heuristic. While the former predicts a boost, the latter predicts no change in the asymmetric dominance effect when there are multiple decoys. Even though their predictions differ, they both agree that the impact should not depend on the type of the target. Thus, neither of them can totally explain our results.

Now we know that the classical asymmetric dominance effect (with a single decoy) has a

(a) Choice Shares Treatment T0

\begin{tabular}{|c|c|c|c|}
\hline treatment $\mathbf{T 0}$ & tasks $1-27$ & $\begin{array}{l}L V L \\
H V L \\
\end{array}$ & $\begin{array}{l}429(56.7 \%) \\
327(43.3 \%)\end{array}$ \\
\hline & tota & & $756(100 \%)$ \\
\hline
\end{tabular}

(b) Choice Shares Treatment $\mathbf{T} \mathbf{1}$

\begin{tabular}{|c||cc|cc|c|}
\hline \multirow{2}{*}{ treatment T1 } & & & \multicolumn{2}{|c|}{ tasks $28-54$} & \\
& & & LVL & HVL & total \\
\hline $\mathcal{X}^{2}(d f=1): 155.9580$ & \multirow{2}{*}{ tasks 1-27 } & LVL & $390(53.2 \%)$ & $\mathbf{1 3 0}(\mathbf{1 7 . 7 \%})$ & $520(70.9 \%)$ \\
$p<0.001$ & & $H V L$ & $54(7.4 \%)$ & $159(21.7 \%)$ & $213(29.1 \%)$ \\
\hline & total & & $444(60.6 \%)$ & $289(39.4 \%)$ & $733(100 \%)$ \\
\hline
\end{tabular}

(c) Choice Shares Treatment T2

\begin{tabular}{|c||cc|cc|c|}
\hline \multirow{2}{*}{ treatment T2 } & & \multicolumn{2}{|c|}{ tasks 28-54 } & \multirow{2}{*|}{ total } \\
\hline $\mathcal{X}^{2}(d f=1): 164.9768$ & \multirow{2}{*}{ tasks 1-27 } & LVL & $390(52 \%)$ & $\mathbf{1 4 9}(\mathbf{1 9 . 9 \%})$ & $539(71.9 \%)$ \\
$p<0.001$ & & $H V L$ & $44(5.9 \%)$ & $167(22.3 \%)$ & $211(28.1 \%)$ \\
\hline & total & $434(57.9 \%)$ & $316(42.1 \%)$ & $750(100 \%)$ \\
\hline \hline
\end{tabular}

(d) Choice Shares Treatment T3

\begin{tabular}{|c||cc|cc|c|}
\hline \multirow{2}{*}{ treatment T3 } & & & \multicolumn{2}{|c|}{ tasks 28-54 } & \\
& & & LVL & HVL & total \\
\hline $\mathcal{X}^{2}(d f=1): 136.9546$ & \multirow{2}{*}{ tasks 1-27 } & LVL & $336(46.3 \%)$ & $\mathbf{1 5 5 ( 2 1 . 4 \% )}$ & $491(67.6 \%)$ \\
$p<0.001$ & & $H V L$ & $52(7.2 \%)$ & $183(25.2 \%)$ & $235(32.4 \%)$ \\
\hline & total & $388(53.4 \%)$ & $338(46.6 \%)$ & $726(100 \%)$ \\
\hline \hline
\end{tabular}

(e) Choice Shares Treatment T4

\begin{tabular}{|c||cc|cc|c|}
\hline \multirow{2}{*}{ treatment T4 } & & & \multicolumn{2}{|c|}{ tasks 28-54 } & \\
& & & $L V L$ & $H V L$ & total \\
\hline $\mathcal{X}^{2}(d f=1): 113.4453$ & \multirow{2}{*}{ tasks 1-27 } & $L V L$ & $362(49.7 \%)$ & $\mathbf{1 5 7}(\mathbf{2 1 . 5 \%})$ & $519(71.2 \%)$ \\
$p<0.001$ & & $H V L$ & $56(7.7 \%)$ & $154(21.1 \%)$ & $210(28.8 \%)$ \\
\hline & total & $418(57.3 \%)$ & $311(42.7 \%)$ & $729(100 \%)$ \\
\hline \hline
\end{tabular}

Note: Numbers in bold correspond to the asymmetric dominance effect

Table 2: Choice Shares across Treatments T0(a)-T4(e) 


\begin{tabular}{|c||l||ll|}
\hline compared treatments & \multicolumn{2}{|c||}{$L V L \mathrm{~s}$ (tasks 1-27) } & \multicolumn{2}{c|}{$H V L \mathrm{~s}$ (tasks 28-54) } \\
\hline T0 versus T1 & $\mathcal{X}^{2}(d f=1): 32.4150, \quad p<0.001$ & $\mathcal{X}^{2}(d f=1): 2.2231, \quad p>0.1$ \\
T0 versus T2 & $\mathcal{X}^{2}(d f=1): 37.9328, \quad p<0.001$ & $\mathcal{X}^{2}(d f=1): 0.2790, \quad p>0.1$ \\
T0 versus T3 & $\mathcal{X}^{2}(d f=1): 18.2146, \quad p<0.001$ & $\mathcal{X}^{2}(d f=1): 1.5067, \quad p>0.1$ \\
T0 versus T4 & $\mathcal{X}^{2}(d f=1): 33.7301, \quad p<0.001$ & $\mathcal{X}^{2}(d f=1): 0.0801, \quad p>0.1$ \\
\hline
\end{tabular}

Table 3: Statistical Test Results for Comparisons of T0 with other Treatments

stronger impact when the target is an $L V L$ than when it is an $H V L$ (Hypothesis 1), and that the effect still exists when there are two decoys (Hypothesis 2(a)). A natural question arises as to whether the type of the target lottery matters also in the case of two decoys. To explore this question, we compare the observations from treatment T0 to those from T3 and T4, separately. As shown in Table 3, the choice share of $L V L \mathrm{~s}$ in treatment T3 is significantly higher than that in T0 (67.6\% versus $\left.56.7 \%, \mathcal{X}^{2}(d f=1): 18.2146, p<0.001\right)$ and the same holds true also for treatment T4 $\left(71.2 \%\right.$ versus $\left.56.7 \%, \mathcal{X}^{2}(d f=1): 33.7301, p<0.001\right)$. On the contrary, the choice share of $H V L$ s does not seem to be significantly different $(46.6 \%$ versus $43.3 \%$ for treatment T3, $\mathcal{X}^{2}(d f=1): 1.5067, p>0.1$, and $42.7 \%$ versus $43.3 \%$ for treatment $\left.\mathbf{T} 4, \mathcal{X}^{2}(d f=1): 1.5067, p>0.1\right)$. Hence, like in the case of a single decoy, the asymmetric dominance effect is stronger when the target is an $L V L$ than when it is an $H V L$, and Hypothesis 3 is supported. Turning now to the last analysis, we explore how the observed asymmetric dominance effect is related to individual risk preferences. First, we group subjects into four categories based on their degree of elicited risk aversion. Then, we examine whether there are significant differences in the asymmetric dominance effect across the different risk categories.

As described in the experimental design, we elicit the subjects' degree of risk aversion by using the binary lottery framework of Dohmen et al. (2010). Subjects are asked to fill out Table 4 deciding in each row whether they prefer a safe option (Option $A$ ) or an all-or-nothing lottery (Option $B$ ). In this table, the payment that Option $A$ yields increases from line to line by 1 Euro. To provide incentives, two subjects in each session were chosen by chance and were then paid for one random decision row of their table, in addition to their earnings from the main experiment.

\begin{tabular}{|l|cc|}
\hline & Option $A$ & Option $B$ \\
\hline 1 & $0 €$ for sure & $50 \%$ chance of winning $30 €$ and $50 \%$ chance of winning $0 €$ \\
\hline 2 & $1 €$ for sure & $50 \%$ chance of winning $30 €$ and $50 \%$ chance of winning $0 €$ \\
\hline 3 & $2 €$ for sure & $50 \%$ chance of winning $30 €$ and $50 \%$ chance of winning $0 €$ \\
\hline$\vdots$ & $\vdots$ & $\vdots$ \\
\hline 20 & $19 €$ for sure & $50 \%$ chance of winning $30 €$ and $50 \%$ chance of winning $0 €$ \\
\hline
\end{tabular}

Table 4: Lottery Framework to Measure the Degree of Risk Aversion

We define 4 risk attitude categories ranging from risk averse, slightly risk averse, risk neutral and risk seeking and use the row where subjects switch once from the lottery (Option $B$ ) to the safe choice (Option $A$ ) as a sorting key to assign subjects to the risk categories. Specifically, if subjects switch before row 10 they are classified as being risk averse. If the switching row is between 11 and 13 (14 and 16), subjects are categorized as slightly risk averse (risk neutral). Finally, subjects switching after row 16 or never are characterized as being risk seeking. Table 5 shows the corresponding distribution of our sorting procedure. Note that subjects, who switch more than once between the options, have been excluded from the sample as their risk pref- 
erences appear to be random. ${ }^{2}$ To make sure that our sorting procedure is correct, we follow Holt and Laury (2002) and Goeree et al. (2003) and apply a maximum likelihood analysis to the binary lottery choices to infer the parameter of relative risk aversion. Consistent with the literature, we find that our estimated parameters fall exactly in intervals that were determined in the literature for assigning subjects to the risk categories defined above. ${ }^{3}$ Thus, we have strong support for our sorting procedure being correctly done.

\begin{tabular}{|l||c|c|c|c|c||c|}
\hline \multicolumn{1}{|c||}{} & \multicolumn{5}{c||}{ treatments } & \\
risk category & T0 & T1 & T2 & T3 & T4 & total \\
\hline risk averse & 7 & 7 & 15 & 11 & 12 & 52 \\
slightly risk averse & 8 & 11 & 8 & 8 & 10 & 45 \\
risk neutral & 7 & 6 & 3 & 4 & 2 & 22 \\
risk seeking & 3 & 3 & 2 & 4 & 1 & 13 \\
\hline total & 25 & 27 & 28 & 27 & 25 & 132 \\
\hline
\end{tabular}

Table 5: Number of Subjects by Risk Attitude Categories

We first consider the control treatment T0 and examine whether the frequency of less risky choices $(L V L)$ increases with the degree of risk aversion. Table 6 shows for each treatment the number of $L V L$ versus $H V L$ choices. Subjects assigned to the risk seeking category choose the less risky lottery $35.80 \%(=29 / 81)$ of the times. This ratio for risk neutral, slightly risk averse and risk averse subjects are $40.74 \%(=77 / 189), 68.06 \%(=147 / 216)$ and $69.31 \%(=131 / 189)$, respectively. To assess the statistical significance of this result, we use the Jonckheere-Terpstra Test for ordered alternatives, which tests the null hypothesis that choice behavior across all the risk attitude categories is statistically the same. The first row of Table 6 shows that the Jonckheere-Terpstra Test for our control treatment T0 yields a $p$-value below 0.1 which provides a strong support that subjects who are more risk averse are choosing the less risky alternative $L V L$ significantly more. Thus, we find first support for consistency between individual risk preferences and choice behavior in the experiment.

\begin{tabular}{|ll||c|c|c|c||cc|}
\hline & & \multicolumn{1}{|c|}{$\begin{array}{c}\text { risk } \\
\text { averse }\end{array}$} & \multicolumn{1}{c|}{$\begin{array}{c}\text { slightly } \\
\text { risk averse }\end{array}$} & $\begin{array}{c}\text { risk } \\
\text { neutral }\end{array}$ & $\begin{array}{c}\text { risk } \\
\text { seeking }\end{array}$ & $\begin{array}{c}\text { J-T test } \\
\text { (z-value, } p \text { ) }\end{array}$ \\
\hline \multirow{2}{*}{ T0 } & choices $1-27$ & 131 vs 58 & 147 vs 69 & 77 vs 112 & 29 vs 52 & 6.93, & $p<0.01$ \\
\hline \multirow{2}{*}{ T1 } & choices $1-27$ & 132 vs 54 & 231 vs 63 & 102 vs 55 & 48 vs 30 & 2.03, & $p<0.05$ \\
& choices $28-54$ & 109 vs 80 & 208 vs 88 & 73 vs 80 & 48 vs 32 & 1.288, & $p<0.1$ \\
\hline \multirow{2}{*}{ T2 } & choices $1-27$ & 339 vs 63 & 134 vs 81 & 51 vs 30 & 17 vs 37 & 8.71, & $p<0.01$ \\
& choices $28-54$ & 299 vs 105 & 95 vs 120 & 31 vs 50 & 13 vs 41 & 9.81, & $p<0.01$ \\
\hline \multirow{2}{*}{ T3 } & choices $1-27$ & 227 vs 54 & 150 vs 63 & 73 vs 34 & 33 vs 71 & 7.85, & $p<0.01$ \\
& choices $28-54$ & 197 vs 99 & 124 vs 92 & 38 vs 69 & 28 vs 76 & 7.67, & $p<0.01$ \\
\hline \multirow{2}{*}{ T4 } & choices $1-27$ & 234 vs 83 & 201 vs 65 & 34 vs 20 & 22 vs 5 & 0.214, & $p>0.1$ \\
& choices $28-54$ & 202 vs 119 & 157 vs 111 & 27 vs 27 & 14 vs 13 & 1.935, & $p<0.05$ \\
\hline
\end{tabular}

Table 6: Lottery Choices by Risk Attitude Categories

We further investigate whether the existence of single and multiple decoys affects this rela-

\footnotetext{
${ }^{2}$ We had to exclude 8 of 140 subjects (5.7\% of the subject sample) from the analysis.

${ }^{3}$ We obtain risk parameters of $r$ equal to 0.567 (risk averse), 0.389 (slightly risk averse), 0.078 (risk neutral) and -0.316 (risk seeking). In fact our classification is consistent with the ranges provided in Holt and Laury (2002) (Table 3) and Goeree et al. (2003) (Table 5) with the exception of those classified as slightly risk averse. Our estimation of $r=0.389$ for slightly risk averse subjects according to their lottery choices is somewhat above the range $0.09<r<0.36$ defined in Goeree et al. (2003) but within the range defined in Holt and Laury (2002): $0.15<r<0.41$.
} 
tionship. Table 6 presents the Jonckheere-Terpstra Test results for the first and the second half of the tasks in treatments T1-T4 separately. With only one exception (the first half of the tasks in treatment T4), we find over all treatments and choice sets similar patterns of consistency: that is, the share of the $L V L$ increases with the degree of risk aversion. As shown in Table 6, this relationship holds true even if the decoy is targeting an $H V L$ in the second half of the tasks. ${ }^{4}$

We deepen our analysis by examining whether the asymmetric dominance effect is moderated by individual risk preferences. With this purpose in mind, we scrutinize the behavior described by the effect across the four risk attitude categories in treatments T1 to T4. Table 7 shows that with only two exceptions (risk seeking subjects in treatments $\mathbf{T} 2$ and $\mathbf{T 4}$ ) the asymmetric dominance effect is significant for every risk attitude category. This indicates that irrespective of their degree of risk aversion, subjects exhibit the asymmetric dominance effect. Additionally, we find with only one exception that the strength of the asymmetric dominance effect does not differ across the risk categories (Jonckheere-Terpstra Test, T1: $z=0.570, p>0.1$; T2: $z=3.567, p<0.01$; T3: $z=0.828, p>0.1$; T4: $z=1.321, p>0.1)$. From these findings we can conclude that the effect in consideration does not depend on the degree of risk aversion and, thus, reject our last hypothesis. ${ }^{5}$

\begin{tabular}{|c|l||c||cc|}
\hline treatment & risk category & asymmetric dominance effect & $\mathcal{X}^{2}$ test $($ value, $p)$ \\
\hline \multirow{4}{*}{ T1 } & risk averse & $36(19.35 \%)$ & $40.1450, \quad p<0.01$ \\
& slightly risk averse & $42(14.33 \%)$ & $72.9781, \quad p<0.01$ \\
& risk neutral & $37(24.67 \%)$ & $19.3511, \quad p<0.01$ \\
& risk seeking & $14(18.18 \%)$ & $6.5208, \quad p<0.01$ \\
\hline \multirow{5}{*}{ T2 } & risk averse & $60(14.96 \%)$ & $79.1587, \quad p<0.01$ \\
& slightly risk averse & $52(24.30 \%)$ & $41.1170, \quad p<0.01$ \\
& risk neutral & $26(32.10 \%)$ & $6.7333, \quad p<0.01$ \\
& risk seeking & $11(20.37 \%)$ & $1.7088, \quad p>0.1$ \\
\hline \multirow{5}{*}{ T3 } & risk averse & $57(20.36 \%)$ & $33.7512, \quad p<0.01$ \\
& slightly risk averse & $41(19.25 \%)$ & $49.0848, \quad p<0.01$ \\
& risk neutral & $37(34.91 \%)$ & $18.4889, \quad p<0.01$ \\
& risk seeking & $16(16.00 \%)$ & $11.7032, \quad p<0.01$ \\
\hline \multirow{3}{*}{$\mathbf{T 4}$} & risk averse & $60(19.05 \%)$ & 47.1843, & $p<0.01$ \\
& slightly risk averse & $64(24.24 \%)$ & $34.1654, \quad p<0.01$ \\
& risk neutral & $7(12.96 \%)$ & $31.7647, \quad p<0.01$ \\
& risk seeking & $10(37.04 \%)$ & 0.3453, & $p>0.1$ \\
\hline
\end{tabular}

Note: As in Table 2 the asymmetric dominance effect is given as the number of observations in which an LVL is chosen in a task from the first half of tasks (1-27) and an HVL is chosen in the corresponding task from the second half (28-54). The percentage is shown in brackets.

Table 7: Observed Asymmetric Dominance Effect by Risk Attitude Category

\footnotetext{
${ }^{4}$ One may argue that the findings might be affected by the relatively low numbers of risk seeking subjects. As a further robustness check, the tests have been repeated after merging the risk neutral and risk seeking categories. The results, available in Table 9 of Appendix B, remain unaltered.

${ }^{5}$ As before, the analysis is repeated after merging the risk neutral and risk seeking categories. The results show that the asymmetric dominance effect is significant for every risk attitude category with no exception and it is still uncorrelated with the degree of risk aversion. This additional analysis can be retrieved in Table 10 of Appendix B.
} 


\section{Discussion}

This experimental study adds to the understanding of the asymmetric dominance effect when choice sets consist of two-outcome lotteries. Our analysis suggests that the impact of a decoy on a low-variance lottery $(L V L)$ is much higher than on a high-variance lottery $(H V L)$ of the same expected value. As far as we are aware of, there are only three experimental studies that consider the asymmetric dominance effect when choice alternatives are two-outcome lotteries: Huber et al. (1982), Wedell (1991) and Herne (1999). None of these studies investigates whether the type of a target alternative (in terms of its relative riskiness) has an impact on the effect. However, the results presented by Herne (1999) seem to be parallel to our finding: the choice share of a target is higher when it is an $L V L$ than when it is an $H V L$. The way the results are presented by Huber et al. (1982) and by Wedell (1991) does not allow to make a similar analysis.

The literature concerning the asymmetric dominance effect considers choice from a set that consists of a target, a competitor and a decoy alternative. Our second contribution to this literature is to show that the choice probability of a target lottery systematically increases also when the choice set includes two decoys. We further show that the inclusion of a secondary decoy lottery boosts the asymmetric dominance effect only if the two decoys of the choice set are $r$-decoys that target an $H V L$. For all the other cases in which the two decoys are of the same type, the asymmetric dominance effect with two decoys is not significantly different than the one in the presence of a single decoy. Hence, the impact of a secondary decoy depends on the types of the target and the decoy lottery. This finding falsifies the predictions of the two prominent explanations from the psychology literature for why the classical asymmetric dominance effect (with a single decoy) is observed. While the range-frequency theory by Parducci (1974) predicts an increase in the effect no matter the type of the target alternative, the dominance heuristic explanation forecasts no impact at all. Furthermore, even though a secondary decoy boosts the effect for when an $H V L$ is the target, it does not suppress the validity of our first result. To be precise; the asymmetric dominance effect with two decoys is stronger when the target is an $L V L$ than when it is an $H V L$ (of the same expected value). However, the fact that this experimental study only used two-outcome lotteries warrants discussion about the generalization of our findings. Further research, particularly in the domains of marketing and consumer behavior, is needed to examine whether these effects prevail outside the domain of abstract and monetary lotteries.

Another contribution of our paper is to investigate the correlation between risk aversion and the asymmetric dominance effect. We find that the effect under investigation does not induce any inconsistency in risk behavior and the trend that the more risk averse subjects are, the more the less risky alternative is chosen, is preserved. One might expect a lower choice distortion in the direction of the target lottery from risk averse subjects, since they are expected to stick more to an $L V L$ even if an $H V L$ is targeted. We find, on the contrary, that the asymmetric dominance effect emerges irrespective of risk attitude and there seems to be no correlation between the size of the effect and the degree of risk aversion. Hence, the presence of decoys subtly make decisionmakers choose against their risk preferences, at least to some extent. One interesting avenue for further research would be to provide further sensitivity analysis around this phenomenon. For example, one can increase the salience about the risky nature of the lotteries. Combined with higher stakes one can provide an even more critical test environment for investigating whether the asymmetric dominance effect still prevails in those extreme settings.

Our research has both practical and theoretical implications. On the practical side, it could potentially be of great help in developing profitable marketing strategies for insurance companies, policy makers or product designers. For example, we see similarities between our lottery design and the risks and returns of investment products and portfolios. Our results suggest that if a designer wants to promote an $L V L$ against an $H V L$ product it would be enough to use a single decoy (of any type) that is dominated by the $L V L$. On the other hand, if an $H V L$ needs to 
be promoted against an $L V L$, including two decoys that extend the probability range into the choice set (two $r$-decoys) works better than considering a single decoy. Especially in this domain it is plausible that the degree of risk aversion is an important determinant for choosing among the different portfolios and products. Drawing on our findings, there is a substantial chance that even risk averse investors might consider products which entail higher risk components if they are targeted by a decoy product. On the theoretical side, our results could be integrated into a behavioral industrial organization model to examine the interaction between principals and agents exhibiting the asymmetric dominance effect. ${ }^{6}$ Such a model could have the potential to answer questions like: "How can a monopolist exploit consumers exhibiting this effect?", "Does market competition mitigate this exploitation?" or "How do firms' exploitative strategies affect welfare?"

\footnotetext{
${ }^{6}$ See e.g. Eliaz and Spiegler (2006), Orhun (2009), Barbos (2010), Eliaz and Spiegler (2011), Sürücü (2016).
} 


\section{References}

Barbos, A. (2010). Context effects: A representation of choices from categories. Journal of Economic Theory, 145(3): 1224-1243.

Bateman, I. J., Munro, A., and Poe, G. L. (2008). Decoy effects in choice experiments and contingent valuation: Asymmetric dominance. Land Economics, 84(1): 115-127.

Bateson, M., Healy, S. D., and Hurly, T. A. (2003). Context-dependent foraging decisions in rufous hummingbirds. Proceedings of the Royal Society of London B: Biological Sciences, 270(1521): 1271-1276.

de Clippel, G. and Eliaz, K. (2012). Reason-based choice: A bargaining rationale for the attraction and compromise effects. Theoretical Economics, 7(1): 125-162.

Dhar, R. and Glazer, R. (1996). Similarity in context: Cognitive representation and violation of preference and perceptual invariance in consumer choice. Organizational Behavior and Human Decision Processes, 67(3): 280-293.

Dohmen, T., Falk, A., Huffman, D., and Sunde, U. (2010). Are risk aversion and impatience related to cognitive ability? The American Economic Review, 100(3): 1238-1260.

Doyle, J. R., O'Connor, D. J., Reynolds, G. M., and Bottomley, P. A. (1999). The robustness of the asymmetrically dominated effect: Buying frames, phantom alternatives, and in-store purchases. Psychology \& Marketing, 16(3): 225-243.

Eliaz, K. and Spiegler, R. (2006). Contracting with diversely naive agents. The Review of Economic Studies, 73(3): 689-714.

Eliaz, K. and Spiegler, R. (2011). On the strategic use of attention grabbers. Theoretical Economics, 6(1): 127-155.

Fischbacher, U. (2007). z-tree: Zurich toolbox for ready-made economic experiments. Experimental Economics, 10(2): 171-178.

Frederick, S. (2005). Cognitive reflection and decision making. The Journal of Economic Perspectives, 19(4):25-42.

Goeree, J. K., Holt, C. A., and Palfrey, T. R. (2003). Risk averse behavior in generalized matching pennies games. Games and Economic Behavior, 45(1): 97-113.

Greiner, B. (2015). Subject pool recruitment procedures: Organizing experiments with ORSEE. Journal of the Economic Science Association, 1(1): 114-125.

Heath, T. B. and Chatterjee, S. (1995). Asymmetric decoy effects on lower-quality versus higherquality brands: Meta-analytic and experimental evidence. Journal of Consumer Research, pages $268-284$.

Hedgcock, W. and Rao, A. R. (2009). Trade-off aversion as an explanation for the attraction effect: A functional magnetic resonance imaging study. Journal of Marketing Research, 46(1): $1-13$.

Herne, K. (1997). Decoy alternatives in policy choices: Asymmetric domination and compromise effects. European Journal of Political Economy, 13(3): 575-589.

Herne, K. (1998). Testing the reference-dependent model: An experiment on asymmetrically dominated reference points. Journal of Behavioral Decision Making, 11(3): 181-192. 
Herne, K. (1999). The effects of decoy gambles on individual choice. Experimental Economics, 2(1): 31-40.

Highhouse, S. (1996). Context-dependent selection: The effects of decoy and phantom job candidates. Organizational Behavior and Human Decision Processes, 65(1): 68-76.

Holt, C. A. and Laury, S. K. (2002). Risk aversion and incentive effects. The American Economic Review, 92(5): 1644-1655.

Huber, J., Payne, J. W., and Puto, C. (1982). Adding asymmetrically dominated alternatives: Violations of regularity and the similarity hypothesis. Journal of Consumer Research, 9(1): 9098.

Latty, T. and Beekman, M. (2011). Irrational decision-making in an amoeboid organism: Transitivity and context-dependent preferences. Proceedings of the Royal Society of London B: Biological Sciences, 278(1703): 307-312.

Lea, A. M. and Ryan, M. J. (2015). Irrationality in mate choice revealed by túngara frogs. Science, 349(6251): 964-966.

Lehmann, D. R. and Pan, Y. (1994). Context effects, new brand entry, and consideration sets. Journal of Marketing Research, pages 364-374.

Lichtenstein, S. and Slovic, P. (1971). Reversals of preference between bids and choices in gambling decisions. Journal of experimental psychology, 89(1): 46-55.

Lleras, J. S., Masatlioglu, Y., Nakajima, D., and Ozbay, E. Y. (2016). When more is less: Limited consideration.

Lombardi, M. (2009). Reason-based choice correspondences. Mathematical Social Sciences, 57(1): 58-66.

Masatlioglu, Y., Nakajima, D., and Ozbay, E. Y. (2016). Revealed attention. In Behavioral Economics of Preferences, Choices, and Happiness, pages 495-522. Springer.

Ok, E. A., Ortoleva, P., and Riella, G. (2014). Revealed (p) reference theory. The American Economic Review, 105(1): 299-321.

Orhun, A. Y. (2009). Optimal product line design when consumers exhibit choice set-dependent preferences. Marketing Science, 28(5): 868-886.

Parducci, A. (1974). Contextual effects: A range-frequency analysis. Handbook of perception, 2: $127-141$.

Schmidt, D., Shupp, R., Walker, J. M., and Ostrom, E. (2003). Playing safe in coordination games: The roles of risk dominance, payoff dominance, and history of play. Games and Economic Behavior, 42(2):281-299.

Schwarzkopf, D. L. (2003). The effects of attraction on investment decisions. The Journal of Behavioral Finance, 4(2): 96-108.

Shafir, S., Waite, T. A., and Smith, B. H. (2002). Context-dependent violations of rational choice in honeybees (apis mellifera) and gray jays (perisoreus canadensis). Behavioral Ecology and Sociobiology, 51(2): 180-187.

Simonson, I. (1989). Choice based on reasons: The case of attraction and compromise effects. Journal of Consumer Research, pages 158-174. 
Simonson, I. and Tversky, A. (1992). Choice in context: Tradeoff contrast and extremeness aversion. Journal of Marketing Research, 29(3): 281-295.

Slovic, P. and Lichtenstein, S. (1983). Preference reversals: A broader perspective. The American Economic Review, 73(4): 596-605.

Straub, P. G. (1995). Risk dominance and coordination failures in static games. The Quarterly Review of Economics and Finance, 35(4):339-363.

Sürücü, O. (2016). Welfare improving discrimination based on cognitive limitations. Research in Economics, 70(4): 608-622.

Wedell, D. H. (1991). Distinguishing among models of contextually induced preference reversals. Journal of Experimental Psychology: Learning, Memory, and Cognition, 17(4): 767-778.

Wedell, D. H. and Pettibone, J. C. (1996). Using judgments to understand decoy effects in choice. Organizational Behavior and Human Decision Processes, 67(3): 326-344. 


\section{A Lotteries}

The lotteries we used for the experiment are listed in Table 8. In the control treatment, subjects face 27 choice sets consisting of two lotteries, which we refer to as baseline lotteries. The expected payoffs for these lotteries within the same choice set are always identical (except possibly for small deviations because of rounding). This implies that one of them is a low-variance lottery $(L V L)$ with a high probability of low winnings and the other is a high-variance lottery $(H V L)$ with a low probability of high winnings. The 27 choice tasks of the control treatment To are given in the second and the third columns of Table 8. In the other treatments, we use the same set of 27 baseline lotteries together with their relevant decoys. Note that since we juxtapose each baseline pairs once to a decoy (two decoys) targeting an $L V L$ and once to a decoy (two decoys) targeting an $H V L$, subjects face 54 different choice tasks. In treatment $\mathbf{T 1}$, we use a single $r$-decoy, hence the tasks of this treatment are of the form $\left\{L V L, H V L, L V L_{r}^{\prime}\right\}$ or $\left\{L V L, H V L, H V L_{r}^{\prime}\right\}$. In treatment $\mathbf{T} \mathbf{2}$, we use a single $f$-decoy, hence the tasks of this treatment are of the form $\left\{L V L, H V L, L V L_{f}^{\prime}\right\}$ or $\left\{L V L, H V L, H V L_{f}^{\prime}\right\}$. In the remaining two treatments $\mathbf{T} 3$ and T4, we use two $r$-decoys and two $f$-decoys, respectively. Hence, the choice tasks of this treatment T3 (T4) are of the form $\left\{L V L, H V L, L V L_{r}^{\prime}, L V L_{r}^{\prime \prime}\right\}$ or $\left\{L V L, H V L, H V L_{r}^{\prime}, H V L_{r}^{\prime \prime}\right\}$ $\left(\left\{L V L, H V L, L V L_{f}^{\prime}, L V L_{f}^{\prime \prime}\right\}\right.$ or $\left.\left\{L V L, H V L, H V L_{f}^{\prime}, H V L_{f}^{\prime \prime}\right\}\right)$.

\begin{tabular}{|c|c|c|c|c|c|c|c|c|c|c|c|}
\hline no. & $L V L$ & $H V L$ & $L V L_{r}^{\prime}$ & $L V L$ & & $H V L_{r}^{\prime}$ & $H V L_{r}^{\prime \prime}$ & $L V L_{f}^{\prime}$ & $L V L_{f}^{\prime \prime}$ & $H V L_{f}^{\prime}$ & $H V L_{f}^{\prime \prime}$ \\
\hline 28 & $40 \% 175$ & $\% 350$ & $40 \% 165$ & $40 \% 1$ & 155 & $15 \% 350$ & $10 \% 350$ & $35 \% 175$ & $30 \% \quad 175$ & $20 \% 340$ & $20 \% 3$ \\
\hline $2, \quad 29$ & \% 156 & $\% 280$ & $45 \% 146$ & $45 \%$ & 136 & $20 \% 280$ & $15 \% 280$ & $40 \% 156$ & $35 \% \quad 156$ & $25 \% 270$ & $25 \% 2$ \\
\hline 30 & $\% 140$ & $\% 233$ & $0 \% 130$ & $50 \% 1$ & 120 & $25 \% 233$ & $20 \% 233$ & $45 \% 140$ & $40 \% 140$ & $30 \% 223$ & 09 \\
\hline 31 & \% 127 & $\% 200$ & $5 \% 117$ & $55 \% 1$ & 107 & $0 \% 200$ & $25 \% 200$ & $50 \% 127$ & $45 \% 1$ & 90 & $35 \%$ \\
\hline 32 & $\% 117$ & $0 \% 175$ & $60 \% 107$ & $60 \%$ & 97 & $35 \% 175$ & $30 \% 175$ & $55 \% 117$ & $50 \% 117$ & $40 \% 165$ & $40 \% 15$ \\
\hline 33 & $\% 108$ & $\% 156$ & $65 \%$ & $65 \%$ & 88 & $40 \% 156$ & $35 \% 156$ & $60 \% 108$ & $55 \% 108$ & $45 \% 146$ & $45 \% 136$ \\
\hline 34 & $\% 100$ & $\% 140$ & $70 \%$ & $70 \%$ & 80 & $45 \% 140$ & $40 \% 140$ & $65 \% 100$ & $60 \% 100$ & $50 \% 1$ & $50 \% 120$ \\
\hline 8, & $75 \% \quad 93$ & $55 \% 127$ & $75 \%$ & $75 \%$ & 73 & $50 \% 127$ & $45 \% 127$ & $70 \% \quad 93$ & $65 \% \quad 93$ & $55 \% 117$ & $55 \%$ \\
\hline 36 & 88 & $\% 117$ & $80 \%$ & $80 \%$ & 68 & $55 \% 1$ & $50 \% 117$ & $\begin{array}{lll}75 \% & 88\end{array}$ & $70 \%$ & 07 & $\begin{array}{ll}60 \% & 97\end{array}$ \\
\hline 10,37 & $0 \% 188$ & $20 \% 375$ & $40 \% 178$ & $40 \% 1$ & 168 & $15 \% 375$ & $10 \% 375$ & $35 \% 188$ & $30 \% 188$ & $20 \% 365$ & $20 \% 355$ \\
\hline 11,38 & $\% 167$ & $\% 300$ & $45 \% 157$ & $45 \% 1$ & 147 & $20 \% 300$ & $15 \% 300$ & $40 \% 167$ & $35 \% 167$ & $25 \% 290$ & $25 \% 280$ \\
\hline 12,39 & 150 & $\% 250$ & & $50 \%$ & 130 & $25 \% 250$ & $20 \% 250$ & $45 \%$ & $40 \%$ & $30 \%$ & $30 \%$ \\
\hline 13,40 & $55 \% 136$ & $35 \% 214$ & $55 \% 126$ & $55 \% 1$ & 116 & $30 \% 214$ & $25 \% 214$ & $50 \% 136$ & $45 \% 136$ & $35 \% 204$ & $35 \% 194$ \\
\hline 14,41 & $\%$ & \% 188 & $60 \%$ & $60 \%$ & 105 & $35 \% 188$ & $30 \% 188$ & $55 \% 125$ & $50 \% 125$ & $40 \% 178$ & $40 \%$ \\
\hline 15,42 & $\mid 65 \% 115$ & $45 \% 167$ & $65 \% 105$ & $65 \%$ & 95 & $40 \% 167$ & $35 \% 167$ & $60 \% 115$ & $55 \% 115$ & $45 \% 157$ & $45 \% 147$ \\
\hline 16,43 & $\% 107$ & $\% 150$ & $70 \% \quad 97$ & $70 \%$ & 87 & $45 \% 150$ & $40 \% 150$ & $65 \% 107$ & $60 \% 107$ & $50 \% 140$ & $50 \% 130$ \\
\hline 17,44 & $\% 100$ & $55 \% 136$ & $75 \%$ & $75 \%$ & 80 & $50 \% 136$ & $45 \% 136$ & $70 \% 100$ & $65 \% 100$ & $55 \% 126$ & $55 \% 116$ \\
\hline 18,45 & $80 \% \quad 94$ & $60 \% 125$ & $80 \% \quad 84$ & $80 \%$ & 74 & $55 \% 125$ & $50 \% 125$ & $75 \% \quad 94$ & $70 \% \quad 94$ & $60 \% 115$ & $60 \% 105$ \\
\hline 19,46 & $\% 200$ & $\% 400$ & $40 \% 190$ & $40 \% 1$ & 180 & $15 \% 400$ & $10 \% 400$ & $35 \% 200$ & $30 \% 200$ & 390 & \\
\hline 20,47 & 6\% 178 & $25 \% 320$ & $45 \% 168$ & $45 \%$ & 158 & $20 \% 320$ & $15 \% 320$ & $40 \% 178$ & $35 \% 178$ & $25 \% 310$ & $25 \% 300$ \\
\hline 21,48 & $\% 160$ & $\% 267$ & $50 \% 150$ & $50 \% 1$ & 140 & $25 \% 267$ & $20 \% 267$ & $45 \% 160$ & $40 \% 160$ & $30 \% 257$ & $30 \% 247$ \\
\hline 22,49 & $5 \% 145$ & $5 \% 229$ & $55 \% 135$ & $55 \% 1$ & 125 & $30 \% 229$ & $25 \% 229$ & $50 \% 145$ & $45 \% 145$ & $35 \% 219$ & $35 \% 209$ \\
\hline 23,50 & $60 \% 133$ & $40 \% 200$ & $60 \% 123$ & $60 \% 1$ & 113 & $35 \% 200$ & $30 \% 200$ & $55 \% 133$ & $50 \% 133$ & $40 \% 190$ & $40 \% 180$ \\
\hline 24,51 & $5 \% 123$ & $5 \% 178$ & $65 \% 113$ & $65 \% 1$ & 103 & $40 \% 178$ & $35 \% 178$ & $60 \% 123$ & $55 \% 123$ & $45 \% 168$ & $45 \% 158$ \\
\hline 25,52 & $70 \% 114$ & $50 \% 160$ & $70 \% 104$ & $70 \%$ & 94 & $45 \% 160$ & $40 \% 160$ & $65 \% 114$ & $60 \% 114$ & $50 \% 150$ & $50 \% 140$ \\
\hline 26,53 & $6 \% 107$ & $55 \% 145$ & $75 \%$ & $75 \%$ & 87 & $50 \% 145$ & $45 \% 145$ & $70 \% 107$ & $65 \% 107$ & $55 \% 135$ & $55 \% 125$ \\
\hline 27,54 & $\% 100$ & $60 \% 133$ & $80 \%$ & $80 \%$ & 80 & $55 \% 133$ & $50 \% 133$ & $75 \% 100$ & $70 \% 100$ & $60 \%$ & $60 \% 113$ \\
\hline
\end{tabular}

Table 8: Lotteries 


\section{B Additional Tables}

Tables 9 and 10 are obtained by merging the two categories of risk neutral and risk seeking.

\begin{tabular}{|ll||c|c|c||cc|}
\hline & & $\begin{array}{c}\text { risk } \\
\text { averse }\end{array}$ & $\begin{array}{c}\text { slightly } \\
\text { risk averse }\end{array}$ & $\begin{array}{c}\text { risk } \\
\text { neutral/seeking }\end{array}$ & $\begin{array}{c}\text { J-T test } \\
\text { (value, } p \text { ) }\end{array}$ \\
\hline \multirow{2}{*}{ T0 } & choices $1-27$ & number of $L V L$ vs number of $H V L$ choices & & \\
\hline \multirow{2}{*}{ T1 } & choices $1-27$ & 131 vs 58 & 147 vs 69 & 106 vs 164 & 6.93, & $p<0.01$ \\
& choices $28-54$ & 109 vs 80 & 231 vs 63 & 150 vs 85 & 1.96, & $p<0.05$ \\
\multirow{2}{*}{ T2 } & choices $1-27$ & 339 vs 63 & 134 vs 81 & 68 vs 67 & 8.45, & $p<0.01$ \\
& choices $28-54$ & 299 vs 105 & 95 vs 120 & 44 vs 91 & 9.72, & $p<0.01$ \\
\hline \multirow{2}{*}{ T3 } & choices 1-27 & 227 vs 54 & 150 vs 63 & 106 vs 105 & 7.08, & $p<0.01$ \\
& choices $28-54$ & 197 vs 99 & 124 vs 92 & 66 vs 115 & 7.57, & $p<0.01$ \\
\hline \multirow{2}{*}{ T4 } & choices 1-27 & 234 vs 83 & 201 vs 65 & 56 vs 25 & 0.283, & $p>0.1$ \\
& choices $28-54$ & 202 vs 119 & 157 vs 111 & 41 vs 40 & 1.943, & $p<0.05$ \\
\hline
\end{tabular}

Table 9: Lottery Choices by Risk Attitude Categories

\begin{tabular}{|c|l||c||cc|}
\hline treatment & risk category & asymmetric dominance effect & $\mathcal{X}^{2}$ test $($ value $p)$ \\
\hline \multirow{3}{*}{ T1 } & risk averse & $36(19.35 \%)$ & $40.1450, \quad p<0.01$ \\
& slightly risk averse & $42(14.33 \%)$ & $72.9781, \quad p<0.01$ \\
& risk neutral/seeking & $51(22.47 \%)$ & $25.0530, \quad p<0.01$ \\
\hline \multirow{3}{*}{ T2 } & risk averse & $60(14.96 \%)$ & $79.1587, \quad p<0.01$ \\
& slightly risk averse & $52(24.30 \%)$ & $41.1170, \quad p<0.01$ \\
& risk neutral/seeking & $37(27.41 \%)$ & $10.5326, \quad p<0.01$ \\
\hline \multirow{2}{*}{ T3 } & risk averse & $57(20.36 \%)$ & $33.7512, \quad p<0.01$ \\
& slightly risk averse & $41(19.25 \%)$ & $49.0848, \quad p<0.01$ \\
& risk neutral/seeking & $53(25.73 \%)$ & $31.6728, \quad p<0.01$ \\
\hline \multirow{2}{*}{ T4 } & risk averse & $60(19.05 \%)$ & $47.1843, \quad p<0.01$ \\
& slightly risk averse & $64(24.24 \%)$ & $34.1654, \quad p<0.01$ \\
& risk neutral/seeking & $17(20.99 \%)$ & $26.2745, \quad p<0.01$ \\
\hline
\end{tabular}

Note: As in Table 2 the asymmetric dominance effect is given as the number of observations in which an LVL is chosen in a task from the first half of tasks (1-27) and an HVL is chosen in the corresponding task from the second half (28-54). The percentage is shown in brackets.

Table 10: Observed Asymmetric Dominance Effect by Risk Attitude Category 


\section{Instructions}

The instructions for all treatments and the questionnaire were originally in German. The English instructions presented here are a translation of the originally used ones.

\section{C.1 Experiment Instructions (English version)}

Note that these are the instructions for treatment $\mathbf{T} 3$ and $\mathbf{T} 4$. The number of lotteries, the notation and the figure have been adapted accordingly for the remaining treatments.

\section{General Instructions}

- During the experiment all payments are stated in the fictitious currency "Taler".

- The experiment today consists of 54 decisions situations.

- In none of your decisions does your payment depend on the decisions of the other participants.

- After the experiment we would like to ask you to fill out a questionnaire. For this you will receive a short instruction as soon as the experiment will have ended. For your responses there is no "right" or "wrong". The answers of the questionnaire have no influence on your payment.

\section{Course of the Experiment}

- In all of the 54 decision situations you are asked to decide between different lotteries.

- In every decision situation there are 4 alternative lotteries available. These are denoted by A, B, $\mathrm{C}$ und D.

- Exemplarily the following lotteries are displayed in one decision situation:

Lottery A

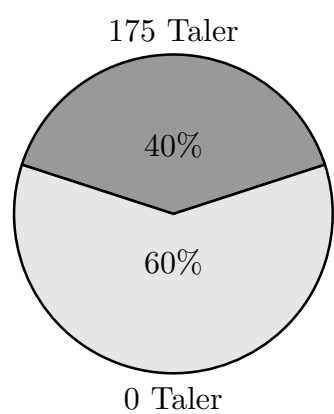

With this lottery you have a $40 \%$ chance to win 175 Taler, nothing otherwise.
Lottery B

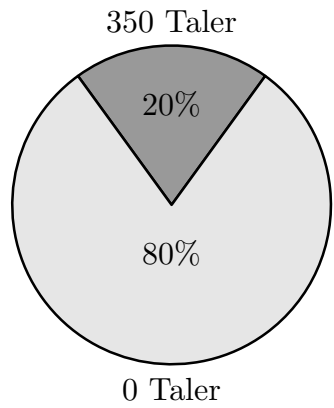

With this lottery you have a $20 \%$ chance to win 350 Taler, nothing otherwise.
Lottery C

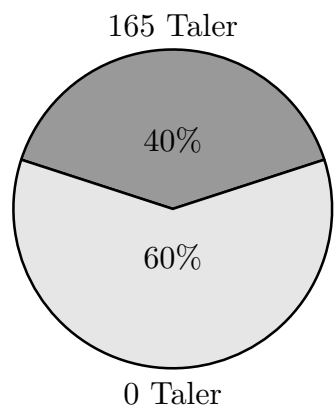

With this lottery you have a $40 \%$ chance to win 165 Taler, nothing otherwise.
Lottery D

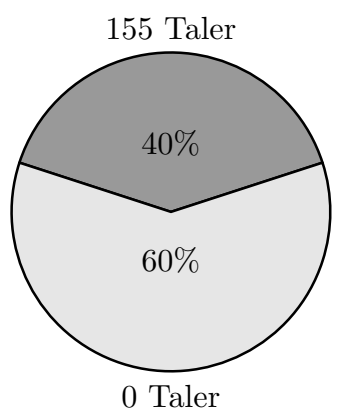

With this lottery you have a $40 \%$ chance to win 155 Taler, nothing otherwise.

To better understand the probabilities of the different lotteries, imagine for lottery A above an urn that contains 100 balls. 40 balls are blue and 60 balls are white. One ball is drawn from this urn. If the ball is blue, you would win 175 Taler and if the ball is white, you would win 0 Taler.

- After you have selected a lottery and confirmed your choice on the screen, the next decision situation starts with the new set of lotteries. 


\section{Payments:}

- Only one decision situation is relevant for your payment.

- After every participant has completed the 54 decision situations, one participant in the room is asked to randomly draw the payment-relevant decision situation. Each of the 54 decision situations is displayed on a card and put in an urn. The chosen participant is asked to draw exactly one of these cards. The card and the related decision situation is for the payment of all participants.

- For your payment, the lottery that you have chosen in this decision situation is played by yourself. To do this you are asked to draw a ball from an urn that contains exactly 100 balls.

- The balls in this urn are either blue or white. The urns are prepared such that the number of blue balls and white balls corresponds to the probabilities of the lottery you selected. If you draw a blue ball, you receive the positive amount in Taler related to that lottery. If you draw a white ball, you receive 0 Taler.

- The payment of your lottery will be converted with an exchange rate of 1 Euro per 20 Taler and will be paid in cash together with a show-up fee of 4 Euro.

\section{Please Note:}

- During the entire experiment, any and all forms of communication are not permitted.

- All mobile phones must be switched off during the complete duration of the experiment.

- The decisions you make within this experiment are anonymous: i.e., none of the other participants gets to know the identity of a person who has made a specific decision.

- Please remain seated until the end of the experiment.

- You will be called forward for your payment by your seat number.

\section{Good luck and thank you very much for your participation in this experiment!}

\section{C.2 Questionnaire Instructions (English version)}

Note that the screenshot included in the questionnaire has been translated in English and is shown schematically.

\section{Questionnaire Instructions}

- The experiment is over now.

\section{Questionnaire Instructions - Part I}

- In part I of the questionnaire we would like to know how you would choose between a safe payment (Alternative A) and a lottery (Alternative B).

- The following screen will be presented to you:

In each row please select if you prefer the safe payment (Alternative A) OR the lottery (Alternative B):

1) Alternative A: $0 €$ for sure $\circ \circ$ Alternative B: $50 \%$ chance of winning $30 €$ and $50 \%$ chance of winning $0 €$.

2) Alternative A: $1 €$ for sure $\circ \circ$ Alternative B: $50 \%$ chance of winning $30 €$ and $50 \%$ chance of winning $0 €$.

3) Alternative A: $2 €$ for sure $\circ \circ$ Alternative B: $50 \%$ chance of winning $30 €$ and $50 \%$ chance of winning $0 €$.

4) Alternative A: $3 €$ for sure $\circ \circ$ Alternative B: $50 \%$ chance of winning $30 €$ and $50 \%$ chance of winning $0 €$.

5) Alternative A: $4 €$ for sure $\circ \circ$ Alternative B: $50 \%$ chance of winning $30 €$ and $50 \%$ chance of winning $0 €$.

6) Alternative A: $5 €$ for sure $\circ \circ$ Alternative B: $50 \%$ chance of winning $30 €$ and $50 \%$ chance of winning $0 €$. 
7) Alternative A: $6 €$ for sure $\circ \circ$ Alternative B: $50 \%$ chance of winning $30 €$ and $50 \%$ chance of winning $0 €$.

8) Alternative A: $7 €$ for sure $\circ$ Alternative B: $50 \%$ chance of winning $30 €$ and $50 \%$ chance of winning $0 €$.

9) Alternative A: $8 €$ for sure $\circ \circ$ Alternative B: $50 \%$ chance of winning $30 €$ and $50 \%$ chance of winning $0 €$.

10) Alternative A: $9 €$ for sure $\circ \circ$ Alternative B: $50 \%$ chance of winning $30 €$ and $50 \%$ chance of winning $0 €$.

11) Alternative A: $10 €$ for sure $\circ \circ$ Alternative B: $50 \%$ chance of winning $30 €$ and $50 \%$ chance of winning $0 €$.

12) Alternative A: $11 €$ for sure $\circ \circ$ Alternative B: $50 \%$ chance of winning $30 €$ and $50 \%$ chance of winning $0 €$.

13) Alternative A: $12 €$ for sure $\circ \circ$ Alternative B: $50 \%$ chance of winning $30 €$ and $50 \%$ chance of winning $0 €$.

14) Alternative A: $13 €$ for sure $\circ \circ$ Alternative B: $50 \%$ chance of winning $30 €$ and $50 \%$ chance of winning $0 €$.

15) Alternative A: $14 €$ for sure $\circ \circ$ Alternative B: $50 \%$ chance of winning $30 €$ and $50 \%$ chance of winning $0 €$.

16) Alternative A: $15 €$ for sure $\circ \circ$ Alternative B: $50 \%$ chance of winning $30 €$ and $50 \%$ chance of winning $0 €$.

17) Alternative A: $16 €$ for sure $\circ \circ$ Alternative B: $50 \%$ chance of winning $30 €$ and $50 \%$ chance of winning $0 €$.

18) Alternative A: $17 €$ for sure $\circ \circ$ Alternative B: $50 \%$ chance of winning $30 €$ and $50 \%$ chance of winning $0 €$.

19) Alternative A: $18 €$ for sure $\circ \circ$ Alternative B: $50 \%$ chance of winning $30 €$ and $50 \%$ chance of winning $0 €$.

20) Alternative A: $19 €$ for sure $\circ \circ$ Alternative B: $50 \%$ chance of winning $30 €$ and $50 \%$ chance of winning $0 €$.

- In each line (from 1 to 20) you have two options:

- A safe payment (Alternative A).

- An "all-or-nothing" lottery in which you can win 30 Euro with a probability of $50 \%$ and nothing with a probability of $50 \%$ (Alternative B).

- For each line please choose either alternative A or B. Mark the left field if you choose Alternative $\mathbf{A}$ or the right field if you choose Alternative B.

\section{Additional profit opportunity in part I of the questionnaire:}

- In the first part of the questionnaire you have another chance to win a payment.

- Two participants in this room will be randomly drawn.

- For the drawing of the two winners, two cards will be randomly drawn from the numbered cards ranging from 1 to 28 .

- The chosen participants will receive their additional payout when all payments are distributed after answering the second part of the questionnaire.

- If you are among the two chosen participants, the additional payment is determined by means of a twenty-sided dice.

- The first draw of this dice determines the line that is payment-relevant.

- If you decided to take Alternative B for the line which will be paid out, you will be asked to cast a twenty-sided dice again. With the numbers 1 to 10 you receive 30 Euro, with 11 to 20 you receive nothing. If you decided to take Alternative A, you will receive the safe payout immediately.

When all participants complete the first part of the questionnaire, part II will follow. The answers in part II are irrelevant for the payout. All questions will be evaluated anonymously and communication is not allowed during the complete experiment.

\section{Thank you very much for your participation in this experiment!}

